\title{
The induction of rat spermatogonial stem cells into neuronal-like cells and behavioral recovery following transplantation in a rat Parkinson's disease model
}

\author{
TE LIU $^{1 *}$, ZHANGBIN GONG $^{2,3 *}$, LENGCHEN HOU $^{4}$ and YONGYI HUANG ${ }^{5}$ \\ ${ }^{1}$ Shanghai Geriatric Institute of Chinese Medicine, Shanghai University of Traditional Chinese Medicine, Shanghai 200031; \\ ${ }^{2}$ Shanghai University of Traditional Chinese Medicine, Shanghai 201203, P.R. China; ${ }^{3}$ College of Agriculture, University \\ of Kentucky, Lexington, KY 40546, USA; ' Department of Anesthesiology, Shanghai 10th People's Hospital, \\ Tongji University School of Medicine, Shanghai 200072, P.R. China; ${ }^{5}$ Laboratoire PROTEE, \\ Bâtiment R, Université du Sud Toulon-Var, 83957 La Garde Cedex, France
}

Received September 1, 2011; Accepted October 21, 2011

DOI: $10.3892 / \mathrm{ijmm} .2011 .828$

\begin{abstract}
Parkinson's disease (PD) is a widespread ageassociated neurodegenerative disorder. Current treatment is symptomatic rather than curative. However, stem cell replacement therapies may have the potential to offer curative treatment. In this study, we demonstrate that rat $\mathrm{CD}_{4} 9 \mathrm{f}^{+}$spermatogonial stem cells (rSSCs) can be induced to become functional dopaminergic neuron-like cells in vitro. Furthermore, when rSSCs were transplanted into 6-hydroxydopamine (6-OHDA)-treated PD rats, the results indicated that rSSCs expressed multiple neuron cell markers and were ameliorative to behavioral recovery in PD rats after induction both in vitro and in vivo. In addition, rSSCs demonstrated increased activity in the regeneration of dopaminergic neuron-like cells, increased migration distances and were associated with improvement in animal behavior in the PD rat model. Therefore, rSSCs could be a source of dopaminergic neuron-like cells with potential benefit in cell replacement therapy for PD.
\end{abstract}

\section{Introduction}

Parkinson's disease (PD) is characterized by a progressive loss of midbrain substantia nigra dopaminergic (DA) neurons that project to the striatum, and these neurons are responsible for the main motor symptoms that characterize the disease $(1,2)$. $\mathrm{PD}$ is a major neurodegenerative disease that affects almost $2 \%$ of the population over the age of 65 years. At present, cell replacement therapies may provide the most promising

Correspondence to: Dr Te Liu, Shanghai Geriatric Institute of Chinese Medicine, Shanghai University of Traditional Chinese Medicine, Shanghai 200031, P.R. China

E-mail: liute79@yahoo.com

*Contributed equally

Key words: cell transplantation, spermatogonial stem cells, Parkinson's disease, rat model prospect for curative treatment (3-7). If cells can be placed in the brain to produce suitable, controlled levels of dopamine release in the striatum, this raises the possibility that the disease process could be attenuated and motor function restored in affected patients.

However, regenerative and cell replacement therapies face several important challenges. Firstly, the main symptoms of PD result from a loss of midbrain DA neurons, but other cell types are also affected. Secondly, a further challenge for fetal tissue grafting is the poor survival of DA neurons following transplantation (1). Previous studies have indicated that not only neural stem cells, but also embryonic stem cells (ESCs) and induced pluripotent stem (iPS) cells can be induced to differentiate into dopaminergic neurons and to provide behavioral improvement in 6-hydroxydopamine (6-OHDA)lesioned rat models (3-10). This suggests that these stem cells have potential for the use of cell therapy in the management of neurodegenerative diseases.

For many years, scientists have been looking for mechanisms that regulate the differentiation of stem cells. The answer is likely to be complex and to involve a variety of contributing factors (9). Despite intensive research over the past decades, little is known about how the self-renewal properties of these stem cells are maintained and how their cell fate is determined $(3,11)$. In addition, some stem cells, such as ESCs, have not been used clinically due to ethical limitations. Indeed, studies have reported that iPS cells, if seeded into the mouse brain, can result in highly malignant teratocarcinomas that do not migrate away from the transplantation site $(6,12-14)$. All of these factors emphasize the importance of establishing safe and effective options.

Spermatogenesis is a highly regulated process in which undifferentiated germ cells classified as spermatogonial stem cells (SSCs) divide and mature to produce spermatozoa during and after puberty. The undifferentiated spermatogonial population in the testes of non-primate mammals consists of type $A_{\text {single }}\left(A_{s}\right), A_{\text {paired }}\left(A_{\text {pr }}\right)$ and $A_{\text {aligned }}\left(A_{a l}\right)$ germ cells (15-18). Traditionally, $A_{s}$ spermatogonia have been considered to be the spermatogonial stem cells. In rodents, $A_{s}$ spermatogonia are 
the stem cells of spermatogenesis, capable of both self-renewal and differentiation (19-22). Previous research demonstrated that cultured SSCs in vitro possess many of the characteristics of true ESCs. SSCs have great potential for cell-based, autologous organ regeneration therapy for various conditions, eliminating the need to use human ESCs.

In this study, we set out to determine which subpopulation of rat $\mathrm{CD} 49 \mathrm{f}^{+}$spermatogonial stem cells (rSSCs) could be induced to differentiate into neuron cell-like cells, particularly dopaminergic neuron cells, and whether rSSCS could influence the speed and effectiveness of dopaminergic neuron cell induction.

\section{Materials and methods}

Preparation of mouse embryonic fibroblasts (MEFs) and human amniotic epithelial cells (hAECs). MEF cells were isolated from 13-day-old C57BL/6 mouse embryos. Cells were mitotically inactivated using mitomycin C (Sigma, St. Louis, MO, USA) as previously described (18).

Rat SSCs isolated and sorted by flow cytometry. The rat SSCs were isolated from 20 SD male rat (Center for Laboratory Animal, Shanghai Institute for Biological and Sciences). Testicular single cell suspensions were collected by a two-step enzymatic digestion and then used for sorting. Briefly, testicular cells were obtained by digestion with $1.0 \mathrm{mg} / \mathrm{ml}$ collagenase IV (Sigma) at $37^{\circ} \mathrm{C}$ for $15 \mathrm{~min}$, followed by digestion with $0.125 \%$ trypsin-1.0 mmol/1 EDTA (Gibco Gaithersburg, MD) at $37^{\circ} \mathrm{C}$ for $10 \mathrm{~min}$. $\alpha 6$-integrin (CD49f) is a component of the laminin receptor expressed by SSCs and can be used for purifying SSCs (18). The dissociated cells $\left(6 \times 10^{6}\right.$ cells $\left./ \mathrm{ml}\right)$ were incubated with fluorescein isothiocyanate (FITC)-conjugated rabbit anti- $\alpha 6$ integrin (CD49f) monoclonal antibody (BD Biosciences) at $4^{\circ} \mathrm{C}$ in the dark for $20 \mathrm{~min}$, and then washed thrice with phosphate-buffered solution (PBS). Next, magnetic-activated cell sorting (MACS) buffer [PBS supplemented with $0.5 \%(\mathrm{w} / \mathrm{v})$ bovine serum albumin and $2.0 \mathrm{mmol} / 1 \mathrm{EDTA}], 6 \times 10^{6}$ cells $/ \mathrm{ml}$ and anti-FITC MACS Microbeads (Miltenyi Biotec, Auburn, CA, USA), were added to the cell pellet. After being mixed, the cells were incubated in the dark at $4^{\circ} \mathrm{C}$ for $15 \mathrm{~min}$. MACS was carried out through the LS-column in a VarioMACS separator (Miltenyi Biotec) following the manufacturer's protocol to enrich for the $\alpha 6$-integrin ${ }^{+}\left(\mathrm{CD} 49 \mathrm{f}^{+}\right)$cells in the suspension. Samples of the cells before and after MACS were analyzed by flow cytometry to determine the expression of $\alpha 6$-integrin ${ }^{+}$ $\left(\mathrm{CD} 49 \mathrm{f}^{+}\right)$to assess the enrichment ratio.

Co-culture of rat SSCs with MEF. Rat SSCs cultures were separated from the feeder cells by treatment with $0.125 \%$ trypsin-EDTA solution and plated onto and co-cultured with MEF. The cells were cultured in DMEM:F12 (1:1) medium supplemented with $15 \%$ KnockOut $^{\mathrm{TM}}$ Serum Replacement, $1 \mathrm{mM}$ sodium pyruvate, $2 \mathrm{mM}$ L-glutamine, $0.1 \mathrm{mM}$ nonessential amino acids, $0.1 \mathrm{mM} \beta$-mercaptoethanol, penicillin $(25 \mathrm{U} / \mathrm{ml})$, streptomycin $(925 \mathrm{mg} / \mathrm{ml})$, and mixed with $10 \mu \mathrm{g} / \mathrm{ml}$ leukemia inhibitory factor (LIF). The cells were incubated in a humidified tissue culture incubator containing $5 \% \mathrm{CO}_{2}$ at $37^{\circ} \mathrm{C}$. All cells had been cultured on the same feeder until passage 2 before using in subsequent experiments.
Alkaline phosphatase staining. Alkaline phosphatase activity of SSCs, which were cultured on hAECs or MEF, or cultured solely without any feeds, were determined using the alkaline phosphatase substrate kit (Sigma-Aldrich, St. Louis, MO, USA) according to the manufacturer's instructions (18).

Flow cytometry analysis for mouse SSCs markers expression. The SSCs, which were cultured on MEF were suspended $\left(6 \times 10^{5}\right.$ cells $\left./ \mathrm{ml}\right)$ and stained with primary antibody against SSCs markers [FITC-conjugated a6-integrin (CD49f, cat\#555736); FITC-conjugated $\beta 1$-integrin (CD29, cat\#558741); FITC-conjugated Thy-1 (CD90, cat\#553004) (all from BD Biosciences); FITC-conjugated Dazl (cat\#ab6717, Abcam)] on ice in Dulbecco's phosphate-buffered saline (DPBS) containing $10 \%$ BSA. Staining was compared with an isotype control antibody (mouse IgG1-FITC, BD Biosciences, Franklin Lakes, NJ, USA) to correct for non-specific binding. Evaluation of antibody staining by flow cytometry and cell sorting by FCM was performed using a FACSVantage SE (BD Biosciences) (23).

Induction of expansion of rSSCs differentiation into neuron cell-like cells in vitro. To induce differentiation into neuron cells, expansion of rSSCs $\left(1 \times 10^{6}\right)$ were seeded in a $10-\mathrm{cm}$ dish and cultured in serum-free DMEM:F12 (1:1) containing $20 \mathrm{ng} / \mathrm{ml}$ EGF and $1 \mathrm{ng} / \mathrm{ml}$ bFGF supplement (all from Gibco), $1 \%$ B27 and N2 supplement (Sigma), and $1.0 \mu \mathrm{mol} / 1$ all-trans retinoic acid (Sigma) for 8 days. All cells were cultured in a humidified incubator, at $37^{\circ} \mathrm{C}$ with $5 \% \mathrm{CO}_{2}$, until $80 \%$ confluent according to the manufacturer's instructions (24).

Immunofluorescence staining and Western blot analysis of neuron cell markers expression. Each group of cells were washed 3 times with FBS and fixed with $4 \%$ paraformaldehyde (Sigma-Aldrich) for $30 \mathrm{~min}$. After blocking, the cells were incubated first with primary rabbit anti-rat neuronal class III $\beta$-tubulin (Tuj1) polyclonal antibody (1:200, Santa Cruz Biotechnology, Inc., Santa Cruz, CA, USA) and rabbit anti-rat neuron-specific enolase (NSE) polyclonal antibody (1:200; Santa Cruz Biotechnology, Inc.) and rabbit anti-rat tyrosine hydroxylase (TH) polyclonal antibody (1:200; Santa Cruz Biotechnology, Inc.) overnight at $4^{\circ} \mathrm{C}$, and then with FITC-conjugated goat anti-rabbit IgG antibody (1:200, Abcam) and $5 \mu \mathrm{g} / \mathrm{ml}$ DAPI (Sigma-Aldrich) at room temperature for $30 \mathrm{~min}$. Then the cells were thoroughly washed with TBST and viewed through a fluorescence microscope (Lecia, DMI3000, Japan).

Protein extracts of each group cells were resolved by $12 \%$ SDS-PAGE and transferred on PVDF (Millipore) membranes. After blocking, the PVDF membranes were washed 4 times for 15 min with TBST at room temperature and incubated with primary antibody. Following extensive washing, membranes were incubated with secondary peroxidase-linked goat antirabbit IgG (1:1,000, Santa Cruz Biotechnology, Inc.) for $1 \mathrm{~h}$. After washing 4 times for $15 \mathrm{~min}$ with TBST at room temperature once more, the immunoreactivity was visualized by enhanced chemiluminescence (ECL kit, Pierce Biotechnology).

The rat model of Parkinson's disease. Adult femal SpragueDawley rats $(n=20)$, weighing between 250-300 g, were obtained from the Shanghai Tongji University with Institutional 
A

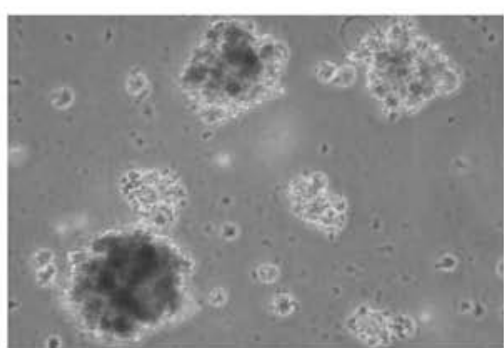

B

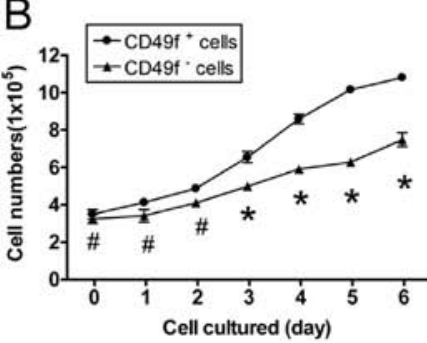

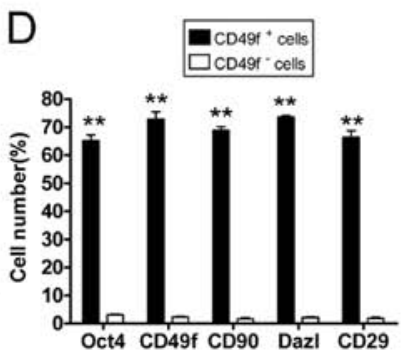

C

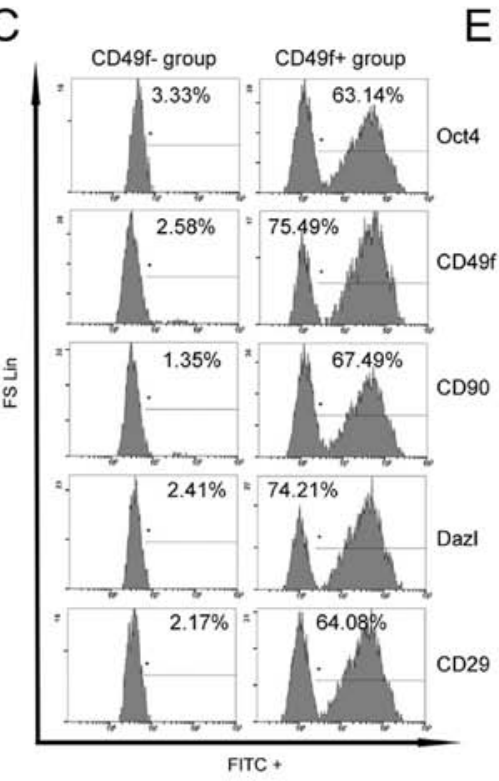

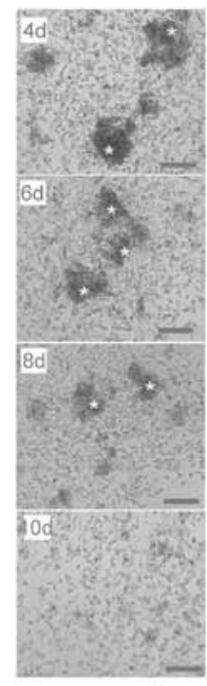

Figure 1. Isolation, enrichment and pluripotency assay of $\mathrm{CD}_{49} \mathrm{f}^{+}$rat spermatogonial stem cells and induction into neuron-like cells. (A) $\mathrm{CD} 49 \mathrm{f}^{+} \mathrm{rSSC}$ sphere clones suspended in medium from FCM sorting. Original magnification, x100. (B) The cells grown with either CD49f ${ }^{+}$or CD49f $\mathrm{fSSC}$ were counted between Day 1 and $6\left({ }^{*} \mathrm{P}<0.05,{ }^{\#} \mathrm{P}>0.05, \mathrm{n}=3\right.$, t-test). (C and D) The SSC marker protein expression between CD49f $\mathrm{f}^{+}$and CD49f rSSCs. Results from the FCM assay

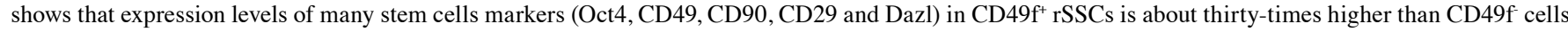
$\left({ }^{* *} \mathrm{P}<0.01, \mathrm{n}=3\right.$, t-test). (E) Induced rSSCs differentiated into neuron-like cells. On the fourth day, we observed rSSC clones and some neuron cell-like cells under microscopy. After 6 days, more and more neuron cell-like cells were observed in the induced group. At 10 days post induction, many neuron cell-like cells were observed in the induced group of rSSCs. Scale bar, $50 \mu \mathrm{m}$.

Aninal Care and Use Committe approval in accordance with institutional guidelines. All rats were maintained from 15 days, 3 to 4 per cage, in a temperature-controlled colony room under standard light-dark cycle with free access to food and water. The study protocol has been previously reported $(4,9,10)$. The animals were divided into two groups: a vehicle group (10 animals) grafted with PBS and an experimental group (10 animals) grafted with rSSCs. All animals received unilateral stereotaxic injections of 6-hydroxydopamine (6-OHDA) (Sigma-Aldrich) into the right medial forebrain bundle (MFB) (AP, $4.4 \mathrm{~mm}$; L, $1.2 \mathrm{~mm}$; DV, $7.8 \mathrm{~mm}$ ) and ventral tegmental area (VTA) (AP, $4.8 \mathrm{~mm}$; L, $1.0 \mathrm{~mm}$; DV, $7.8 \mathrm{~mm}$ ) in order to induce lesions. The anaesthetized animals were injected unilaterally in the intrastriatal region with 6-OHDA using a $10 \mu$ Hamilton microsyringe. Upon completion of the injection, the needle was left in place for an additional $5 \mathrm{~min}$ and then withdrawn at a rate of $1 \mathrm{~mm} / \mathrm{min}$. The rotation behavior of all rats model of PD were evaluated, and the rat models were selected for experiments if the testing result showed a rate of over 6 turns $/ \mathrm{min}$ in response to $0.5 \mathrm{mg} / \mathrm{kg}$ of apomorphine (Sigma-Aldrich) after 4 weeks.

Cell transplantation. Four weeks after surgery, each experimental model received an injection of $5 \mu \mathrm{l}$ of $\mathrm{rSSCs}\left(1 \times 10^{5}\right.$ cell spheres $/ \mu 1)$, which was stained by the membrane probe 1,1'-dioctadecyl-3,3,3',3'-tetramethylindocarbocyanine perchlorate (Dil; Beyotime, Haimen, China) or PBS into the right MFB and VTA, use a $10 \mu \mathrm{l}$ Hamilton microsyringe. A 5-min waiting period allowed the cells to settle before the needle was removed.

Behavioral assay and rotational test. We used the method originally descirbed by Fathi et al (10) and Wen et al $(4,9)$. The rotational behavior of the animals in both groups was evaluated on 1, 3, 5 and 7 weeks after transplantation. In this test, the animals were allowed to habituate for $5 \mathrm{~min}$ in a cylindrical container with $33 \mathrm{~cm}$ diameter and $35 \mathrm{~cm}$ high. After the injection of $0.5 \mathrm{mg} / \mathrm{kg}$ (i.p.) apomorphine hydrochloride (SigmaAldrich), full contralateral rotations were counted for $30 \mathrm{~min}$ in a quiet isolated room.

Statistical analysis. Each experiment was performed as least three times and the data are shown as the mean \pm SE. The differences were evaluated using Student's t-tests. The probability of $<0.05$ was considered to be statistically significant.

\section{Results}

Using flow cytometry, the CD49f $\mathrm{f}^{+} \mathrm{rSSCs}$ were sorted and isolated. Under light microscopy, we found that the colonies of rSSCs cultured on mouse embryonic fibroblasts (MEFs) appeared to be more isolated and rounded, which was consistent with the presence of more undifferentiated cells (Fig. 1).

rSSCs express specific markers of stem cells. Since alkaline phosphatase (AP) levels decrease as stem cells lose their pluripotency and they begin to differentiate, we measured the AP activity of rSSCs from different subpopulations (CD49f ${ }^{+}$ or CD49f cells). Using microscopy, the deep blue color of AP staining was visible in the CD49f $\mathrm{f}^{+} \mathrm{SSCs}$, but not the CD49f cells (Fig. 2). Thus, the AP activity of the CD $49 \mathrm{f}^{+}$rSSCs was higher than that of the CD49f- cells.

We also analyzed the expression of several stem cell markers by FCM (Fig. 1). The expression of many stem cell markers (Oct4, CD49f, CD29, CD90 and Dazl) in the CD49f ${ }^{+}$ 
A

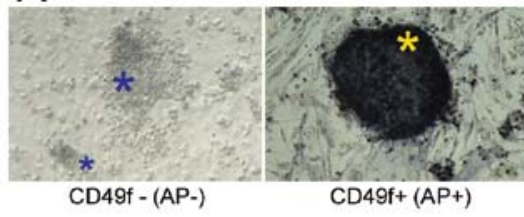

C Non-induced group
B

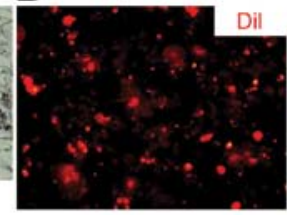

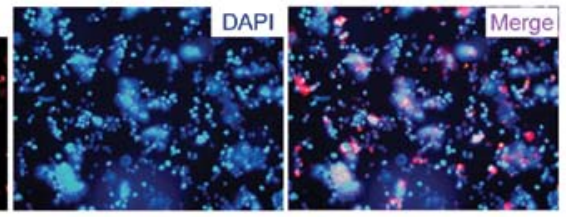

Induced group

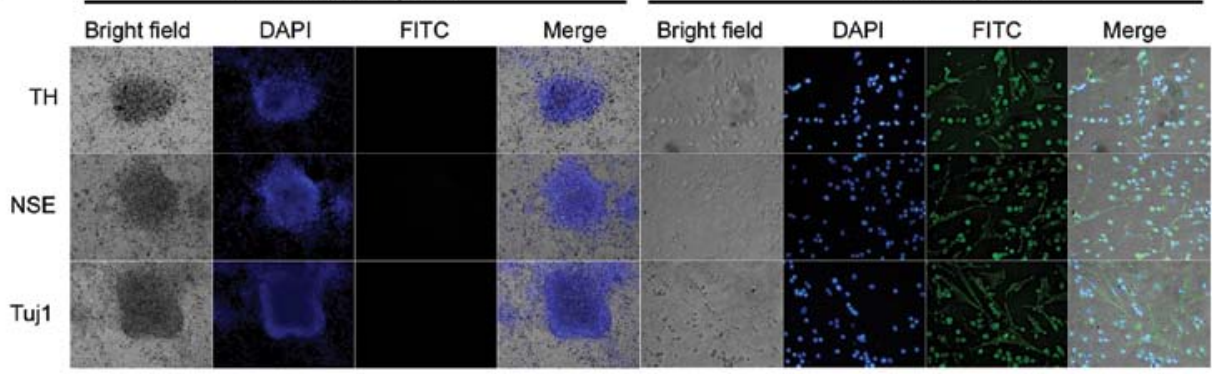

D

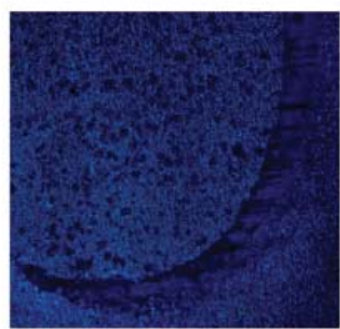

Vehicle group

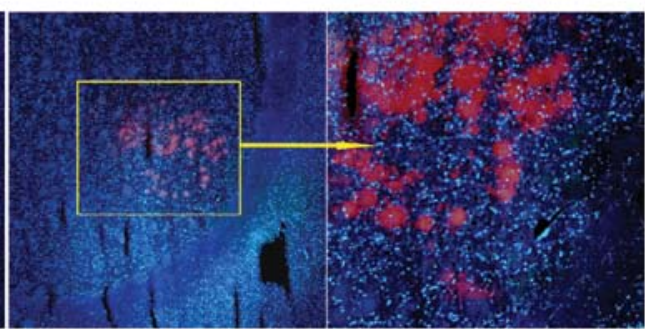

rSSCs-treated group

Figure 2. Expression of neuron-specific factors in dopaminergic neuron-like cells on rSSCs following induction in vitro and in vivo. (A) Alkaline phosphatase (AP) staining assay of $\mathrm{CD}_{4} 4 \mathrm{f}^{+}$and CD49f rSSCs. A small number of AP-positive cells were present in the CD49f cells, but many AP-positive cells were found in the CD49f ${ }^{+}$rSSCs. *, AP-positive cells on the mouse embryonic fibroblasts (MEF) feeder layers. Original magnification, x200. (B) The rSSCs were stained by the membrane probe Dil (1,1'-dioctadecyl-3,3,3',3'-tetramethylindocarbocyanine perchlorate). (C) Neuronal class III $\beta$-tubulin (Tuj1), tyrosine hydroxylase (TH) and neuron-specific enolase (NSE) expression in dopaminergic neuron-like cells in the induced group and non-induced group of rSSCs. In the non-induced group, neither TH, Tuj1 nor NSE were expressed as assayed by immunofluorescence (FITC, green). The intensity of Tuj1, NSE and TH staining was higher in the induced group than in the non-induced group. Original magnification, x200. (D) Analysis of survival and differentiation of transplanted rSSCs or PBS in vivo. The results of the immunofluorescence assay for the Dil probe demonstrated that Dil was expressed highly on rSSCs transplanted in the Parkinson's disease (PD) rat model brain, and they survived for at least 4 weeks in vivo. However, the vehicle group could not be found along the injection tract in the PD rat brain. This assay indicated that grafted rSSCs survived and differentiated into dopaminergic neurons at the ventral tegmental area (VTA) and in the medial forebrain bundle (MFB) regions of injured rats. 4',6-diamidino-2-phenylindole (DAPI, blue) was used to stain cellular DNA. Original magnification, $x 40$.

rSSCs was remarkably higher than that observed in the CD49f cells (Fig. 1). In addition, the proliferation rate of $\mathrm{CD}_{4} 9 \mathrm{f}^{+}$ rSSCs was also higher than that of the CD49f cells (Fig. 1). In summary, we found that $\mathrm{CD}_{4} 9 \mathrm{f}^{+} \mathrm{rSSC}$ could be sorted using FCM expression of strong pluripotency.

rSSCs express neuron markers following induction. Firstly, rSSCs were divided into two groups: the first was cultured in the neuron-induced medium (induced group); the others were cultured in SSCs basic medium (non-induced group). Following the commencement of induction, on Day 4, rSSC clones and some neuron cell-like cells were visible under the microscope (Fig. 1). After 6 days, increasing numbers of neuron cell-like cells could be seen in the induced group. However, the boundary of rSSCs in this group was less clear than that of the non-induced group. Ten days post-induction, many neuron cell-like cells could be seen in the induced group of rSSCs. However, few cells of this type could be found in the non-induced group. To determine whether these cells were neuronal cells, cell markers were assayed using immunofluorescence (IF) staining and Western blotting. IF staining was performed 10 days after induction to compare the expression levels of the neuron-specific enolase (NSE) and Tuj1 in the cells of each group. Expression levels of both proteins were increased in the induced group compared to the non-induced group (Fig. 2).

In addition, we immunostained the cells in each group with rat-specific anti-tyrosine hydroxylase (TH) antibodies in order to determine the pattern of differentiation of rSSCs in vitro. TH-positive cells were observed in the induced group. There were no positive cells in the non-induced group. These results suggest that rSSCs have a great pluripotential capacity, and can easily differentiate into neuronal-like cells by induction in vitro. Indeed, following induction, these rSSCs expressed the dopaminergic neuron marker $\mathrm{TH}$, and may retain the potential to differentiate into dopaminergic neurons. In addition, Western blotting revealed that levels of Tuj1, NSE and TH proteins were $98.59 \pm 1.33 \%, 89.17 \pm 2.06 \%$ and $39.93 \pm 1.68 \%$, respectively, compared with control glyceraldehyde 3-phosphate dehydrogenase (GAPDH) expression levels in the induced group (Fig. 3). These values were significantly higher than those observed in the non-induced group $(8.73 \pm 1.58 \%$, 

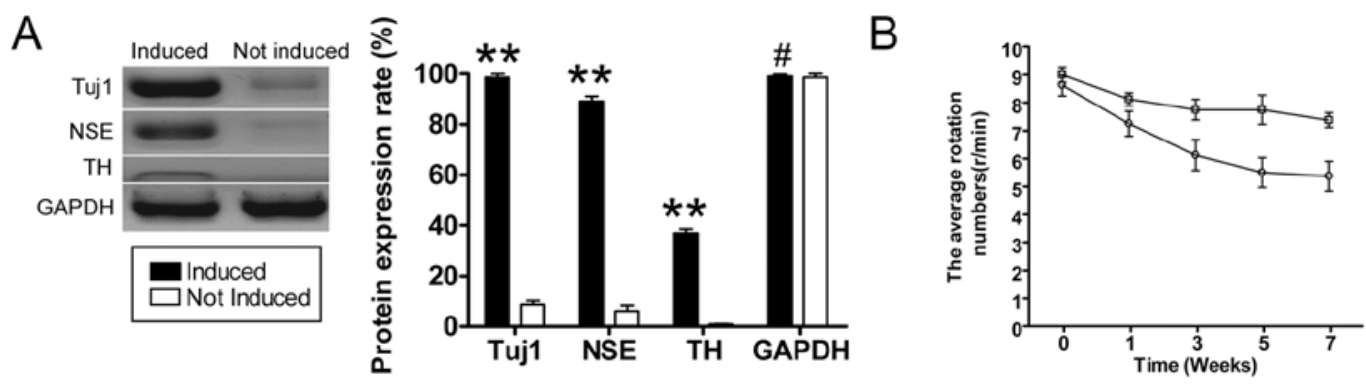

Figure 3. Analysis of the expression of neuron markers by Western blotting assay and behavioral analysis of a PD rat model by apomorphine-induced rotations after transplantation of rSSCs or PBS. (A) Protein expression of Tuj1, NSE and TH was assessed by Western blotting and compared between the induced and the non-induced groups. In the induced group, levels of Tuj1, TH and NSE were significantly higher than those of the non-induced group. ${ }^{* *} \mathrm{P}<0.01 ;{ }^{*} \mathrm{P}>0.05$, $\mathrm{n}=3$, t-test. (B) Apomorphine-induced rotations decreased significantly in the rSSC transplantation group compared with the vehicle group $(\mathrm{P}<0.05$, $\mathrm{n}=8)$. This result suggests that rSSCs can aid recovery.

$6.13 \pm 2.25 \%$ and $0.91 \pm 0.13 \%$ of GAPDH levels, respectively). These results indicate that Tuj1 and NSE are abundant and highly expressed in rSSCs during the induction of neuron cell differentiation.

Survival, migration and differentiation of transplanted rSSCs in the PD rat model brain. The brain injection tracts were examined to confirm the presence of transplanted rSSCs, which were stained using the membrane probe Dil. Dil-probe cells were transplanted and were observed for 4 consecutive weeks. As anticipated, we found that Dil-probe rSSCs could be found along the injection tract in the PD rat model brain (Fig. 2), which demonstrated that the transplanted SSCs can survive within the PD rat brain for at least 4 weeks in vivo, and morphological changes can be seen after this period. However, the vehicle group could not be found along the injection tract in the PD rat brain, which reinforced the observation that the grafted rSSCs survived.

For cell migration, we found that the rSSCs migrated from the graft for 4 consecutive weeks after transplantation (Fig. 2). Dil-probe-transduced SSCs revealed that the migrated cells were present in the PD rat brain for 4 consecutive weeks. When the insets were enlarged, the neuron-like cells were detected 4 weeks later in the vicinity of the infarct area. There were few positive cells in the PBS-treated PD rats (vehicle group). These results suggest that SSC migrate in the PD rat brain.

Stimulation of $r$ SSCs to behavioral and functional recovery in a PD rat model. To assess the functional effects of rSSCs or PBS transplantation on a rat PD model, the animals were tested for rotational behavior over $30 \mathrm{~min}$ following intraperitoneal injection of apomorphine (Fig. 3). Apomorphine-induced rotations decreased significantly in the SSC transplantation group compared with the vehicle group $(\mathrm{P}<0.05, \mathrm{n}=8)$. Furthermore, in the SSC transplantation group, there was a significant difference in rotational behavior between different weeks $(\mathrm{P}<0.05$, $\mathrm{n}=8$ ). These results suggest that treatment with SSCs is a more effective approach, and that SSCs can have a favorable effect on behavioral and functional recovery in the rat PD model.

\section{Discussion}

To date, therapeutic approaches for PD provide symptomatic relief but fail to change the course of the disease. Therefore, there is a clear need for regenerative and cell replacement therapies (1). At present, laboratory-based cell replacement therapies may provide the most promising prospect for curative treatment. The question remains as to which stem cells may have the greatest potential for dopamine neuron replacement in PD. At present it appears that ESCs, iPS cells and NSCs demonstrate the greatest efficiency in generating functional midbrain dopaminergic neurons $(3,5-7)$. However, safety aspects must also be considered. It is not ethically acceptable to inject cells into humans when they may retain the potential to divide in vivo, and there is therefore, a need to generate a cell source that is free from proliferating cells (5). As demonstrated in previous reports, cultured SSCs in vitro possess many of the characteristics of true ESCs (19). Furthermore, these cells only express low levels of major histocompatibility complex class I antigens and are more easily accessible than other adult stem cells, making them a potential autologous donor source for stem cell therapy. Therefore, we set out to determine which SSCs have the potential to differentiate into neuron cell-like cells, and whether these cells could influence the speed and effectiveness of dopaminergic neuron cell induction.

Firstly, we isolated and enriched $\mathrm{CD}_{49 \mathrm{f}^{+}}$cells from rat testicles and confirmed that they were spermatogonial stem cells and pluripotent, which was consistent with previous reports. Induced differentiation activated a number of genes related to dopaminergic neuron cell development and function in rSSCs, which indicated that these cells have the capacity to differentiate as dopaminergic neuron cells with neuron factors-sensing ability. To prove that rSSCs can be induced to differentiate into neuron cell-like cells, including dopaminergic neuron cells, and that those cells play an important role in this process, neuron cell markers were detected by Western blotting and IF. We found that neuronal cell markers, including dopaminergic neuron markers, were more highly expressed in the induced group of rSSCs than in the non-induced group, which indicated that rSSCs are indeed important in this process. Furthermore, rSSCs or PBS were transplanted into 6-OHDA-treated PD rats. The results from this approach indicate that rat SSCs can differentiate into neurons in the ventral tegmental area (VTA) and medial forebrain bundle (MFB), and migrate around the lesion site. In addition, these cells were ameliorative to behavioral recovery in the PD rats in vivo. These observations suggest that tissue reconstruction produced by the transplanted rSSCs is necessary prior to 
functional recovery. Such a tissue repair mechanism is most likely required for stable recovery. Potential problems with rSSCs therapy include the development of techniques that can promote better graft survival.

In conclusion, we have provided insight into how rSSCs and brain tissue interact with each other to promote recovery in a rat PD model. Analysis of the transplanted cells and the host tissue indicate that rSSCs play a critical role in cell survival, migration and differentiation into neuron cells, including dopaminergic neurons. Furthermore, behavioral tests demonstrated that rSSCs can significantly improve injury repair. Based on this data, we conclude that rSSCs can be induced to differentiate into cells with the function and characteristics of neuron cells, particularly dopaminergic neurons, and that they could be used as a neuron-replacement population. Combined cell therapy and pharmacological treatment at cellular and organismal levels has the potential to provide an in-depth understanding of both theoretical mechanisms and clinical applications in PD recovery.

\section{Acknowledgements}

This study was supported by a grant from the National Natural Science Foundation of China (no. 81001565) to Z.G. and T.L., the Shanghai Committee Medical Science Foundation of China (no. 10411967100) and the Natural Science Foundation of School of Medicine of Shanghai Jiaotong University (no. YZ1046) to T.L.

\section{References}

1. Arenas E: Towards stem cell replacement therapies for Parkinson's disease. Biochem Biophys Res Commun 396: 152-156, 2010.

2. Tonnesen J, Parish CL, Sorensen AT, et al: Functional integration of grafted neural stem cell-derived dopaminergic neurons monitored by optogenetics in an in vitro Parkinson model. PLoS One 6: e17560, 2011.

3. Kim HJ: Stem cell potential in Parkinson's disease and molecular factors for the generation of dopamine neurons. Biochim Biophys Acta 1812: 1-11, 2011.

4. Gu S, Huang H, Bi J, Yao Y and Wen T: Combined treatment of neurotrophin-3 gene and neural stem cells is ameliorative to behavior recovery of Parkinson's disease rat model. Brain Res 1257: 1-9, 2009.

5. Fricker-Gates RA and Gates MA: Stem cell-derived dopamine neurons for brain repair in Parkinson's disease. Regen Med 5: 267-278, 2010.

6. Luo Y, Kuang SY and Hoffer B: How useful are stem cells in PD therapy? Parkinsonism Relat Disord 15 (Suppl 3): S171-S175, 2009.
7. Schwarz SC and Schwarz J: Translation of stem cell therapy for neurological diseases. Transl Res 156: 155-160, 2010.

8. Zhang L, Gu S, Zhao C and Wen T: Combined treatment of neurotrophin-3 gene and neural stem cells is propitious to functional recovery after spinal cord injury. Cell Transplant 16: 475-481, 2007.

9. Wang L, Wang J, Wu Y, Pang S, Pan R and Wen T: A novel function of dcf1 during the differentiation of neural stem cells in vitro. Cell Mol Neurobiol 28: 887-894, 2008.

10. Fathi F, Altiraihi T, Mowla SJ and Movahedin M: Transplantation of retinoic acid treated murine embryonic stem cells and behavioural deficit in Parkinsonian rats. Indian J Med Res 131: 536-544, 2010.

11. Shi Y, Sun G, Zhao C and Stewart R: Neural stem cell selfrenewal. Crit Rev Oncol Hematol 65: 43-53, 2008.

12. Bjorklund LM, Sanchez-Pernaute R, Chung S, et al: Embryonic stem cells develop into functional dopaminergic neurons after transplantation in a Parkinson rat model. Proc Natl Acad Sci USA 99: 2344-2349, 2002.

13. Hoehn M, Kustermann E, Blunk J, et al: Monitoring of implanted stem cell migration in vivo: a highly resolved in vivo magnetic resonance imaging investigation of experimental stroke in rat. Proc Natl Acad Sci USA 99: 16267-16272, 2002.

14. Chen L, He DM and Zhang Y: The differentiation of human placenta-derived mesenchymal stem cells into dopaminergic cells in vitro. Cell Mol Biol Lett 14: 528-536, 2009.

15. Chen JX, Xu LL, Wang XC, Qin HY and Wang JL: Involvement of c-Src/STAT3 signal in EGF-induced proliferation of rat spermatogonial stem cells. Mol Cell Biochem: June 17, 2011 (Epub ahead of print).

16. Mok KW, Mruk DD, Lee WM and Cheng CY: Spermatogonial stem cells alone are not sufficient to re-initiate spermatogenesis in the rat testis following adjudin-induced infertility. Int J Androl: June 22, 2011 (Epub ahead of print).

17. Oatley JM, Kaucher AV, Avarbock MR and Brinster RL: Regulation of mouse spermatogonial stem cell differentiation by STAT3 signaling. Biol Reprod 83: 427-433, 2010.

18. Liu T, Guo L, Liu Z and Cheng W: Human amniotic epithelial cells maintain mouse spermatogonial stem cells in an undifferentiated state due to high leukemia inhibitor factor (LIF) expression. In Vitro Cell Dev Biol Anim 47: 318-326, 2011.

19. Dym M, He Z, Jiang J, Pant D and Kokkinaki M: Spermatogonial stem cells: unlimited potential. Reprod Fertil Dev 21: 15-21, 2009.

20. de Rooij DG: Proliferation and differentiation of spermatogonial stem cells. Reproduction 121: 347-354, 2001.

21. Maki CB,Pacchiarotti J, Ramos T, et al: Phenotypic and molecular characterization of spermatogonial stem cells in adult primate testes. Hum Reprod 24: 1480-1491, 2009.

22. Niu Z, Goodyear SM, Rao S, et al: MicroRNA-21 regulates the self-renewal of mouse spermatogonial stem cells. Proc Natl Acad Sci USA 108: 12740-12745, 2011.

23. Hermann BP, Sukhwani M, Simorangkir DR, Chu T, Plant TM and Orwig KE: Molecular dissection of the male germ cell lineage identifies putative spermatogonial stem cells in rhesus macaques. Hum Reprod 24: 1704-1716, 2009.

24. Liu T, Guo L, Liu Z, Huang Y and Cheng W: Induction of dopaminergic neuronal-like cells from $\mathrm{CD} 44^{+}$human amniotic fluids that are ameliorative to behavioral recovery in a Parkinson's disease rat model. Int J Mol Med 28: 745-752, 2011. 